\section{Severe Motor Polyneuropathy Secondary to the Use of Vincristine in a Patient with Acute Lymphoblastic Leukaemia in the Period of April 2013}

\section{Abstract}

Describe the severe motor polyneuropathy secondary to the use of Vincristine in a patient with acute lymphoblastic leukemia in the period of April 2013.

Specific objectives:

1. Determine the sociodemographic characteristics of patients in study.

2. Identify the factors that favoured the onset of motor polyneuropathy secondary to the use of vincristine in the patient study.

3. To determine the clinical manifestations of the patient.

4. To describe the electrophysiological changes secondary motor polyneuropathy the use of vincristine in the patient study.

Keywords: Polyneuropathy; Vincristine; Patient; Lymphoblastic; Leukaemia

\section{Cristhian Berrios}

San Juan De Dios Esteli Hospital, Nicaragua

Corresponding author: Cristhian Berrios

\section{drchristberrmai@yahoo.com}

San Juan De Dios Esteli Hospital, Nicaragua.

Tel: +50587787657

Citation: Berrios C. Severe Motor Polyneuropathy Secondary to the Use of Vincristine in a Patient with Acute Lymphoblastic Leukaemia in the Period of April 2013. J Pediatr Care. 2016, 3:1.

Received: October 24, 2016; Accepted: December 24,2016 ; Published: December 30, 2016

\section{Introduction}

Acute lymphoblastic leukemia is a malignancy characterized by uncontrolled lymphoid blood cells and is the most common malignancy in children, meaning around three quarters of all newly diagnosed leukemias proliferation. In our country, it is the most common tumor, meaning $36.5 \%$ of all cancers. The peak incidence occurs between 3 to 4 years old.

There are different treatment modalities, and depends on the response and the severity of the disease, where possible decrease neuropathy; although it produces a long-term physical disability which limits the basic activities of daily living, which hinders rapid reintegration of the patient to their social environment. Within the drugs used to treat patients with acute lymphoblastic leukemia is vincristine, drug whose effect is the most feared disease of the peripheral nerves; by relying this time and dose which is directly related to the damage, which is evident both clinically and electro physiologically. Measurable and practical for information on nerve function, in order to observe the pathophysiological changes that occur in patients with neuropathy, noninvasive, objective, measuring speeds neuro-conduction.

\section{Theoretical Framework}

Cancer is characterized by the existence of cells that have undergone a change in the control mechanisms that regulate their differentiation ability and proliferation. Excessive proliferation causes penetration into adjacent tissues, compression of adjacent structures (nerves, vessels, etc.) and migration to other areas where they maintain their ability to grow and proliferate. The ultimate goal of the anticancer therapy is the complete elimination of any cancer cells through surgery, radiotherapy and pharmacological methods. Vincristine is within drugs that bind to tubulin and belongs to the vinca alkaloids [1].

\section{Chemical characteristics}

Vinblastine vincristine and are obtained by two natural alkaloids of Vinca rosea purification plant. Vindesine is a semisynthetic analogue, desacetyl vinblastine carboxamide, initially identified as metabolite vinblastine. They consist of two rings of similar structure, vindoline and catharanthine, united by a bridge of two carbons. The differences between the three molecules are located in the catharanthine and, although very few, endow each of a characteristic different antitumor spectrum and pharmacological properties [2]. 


\section{Mechanism of Action}

Alkaloids penetrate the cells thanks to a conveyor system that may be common to other macromolecules such antitumor antibiotics. Within cells interact specifically with tubulin by specific association with two protein dimers consisting molecule. Consequently, tubulin cannot polymerize to form microtubules involved in various cellular functions, among which the mitotic spindle formation, displacement of transmitters in axons, etc. Microtubule structures undergo a process of increasing disintegration [3].

The (though perhaps not unique) most obvious effect and primarily responsible for the antitumor action is the arrest of mitosis in metaphase, with dispersion and disorganization of chromosomal material. The time of maximum cell sensitivity to exposure Vinca alkaloids is the last or late phase of the phase $M$. Cellular resistance may be due to inability to penetrate alkaloids cells deficiency of the conveyor system or to a decrease in fixability tubulin [4].

\section{Pharmacokinetics}

They are poorly absorbed from the gastrointestinal tract. The three alkaloids show a three-compartment kinetics, with halflives of 0.8 plasma $7.4164 \mathrm{~min}$ and for vincristine; 3.9, 53 and $1200 \mathrm{~min}$ to vinblastine, and 3.9, 99 and $1200 \mathrm{~min}$ to vindesine. Vincristine, and vinblastine accumulate in platelets, they are extensively metabolized in the liver and the metabolites are excreted bile and feces; by the kidney are removed in less than $15 \%$. Vindesine appears showing a similar pattern of elimination. Vincristine passes the blood brain barrier but reaches much lower than plasma concentrations [5].

\section{Adverse reactions}

Before administering the drug should inform patients and/or their relatives or guardians of the possible occurrence of adverse reactions. In general, adverse reactions are reversible and doserelated. The most common adverse reaction is hair loss; the most annoying reactions are neuromuscular origin.

When single weekly dose of this medicine are used, adverse reactions appear in the form of leukopenia, neuralgia and constipation but usually are of short duration (e.g. less than seven days). When doses are reduced, these reactions may lessen or disappear. The severity of these reactions seems to increase when the adjusted amount of the drug is administered in divided doses. Other adverse reactions, such as hair loss, sensory loss, numbness, difficulty walking, ataxic gait, deep tendon reflexes and muscle atrophy may persist for at least the duration of treatment [6].

Generalized sensorimotor dysfunction may become progressively severe with continued treatment. Although in most cases these symptoms disappear at about the sixth week of treatment discontinuation in some patient's neuromuscular disorders may persist for a long time. During maintenance therapy can grow hair again.

Hypersensitivity: Isolated cases of allergic reactions, such as anaphylaxis, eczema and edema, in patients receiving chemotherapy with several drugs, including vincristine sulfate was.

Gastrointestinal: Constipation, abdominal cramps, weight loss, nausea, vomiting, oral mucositis, diarrhea, paralytic ileus, necrosis and/or intestinal perforation, and anorexia. Constipation can occur as obstruction in proximal segments of the colon, and if a physical examination is performed rectum may appear empty. The presence of abdominal cramping pain with an empty rectum may mislead the physician. To confirm these tables is useful to an $x$-ray of the abdomen with the patient lying down. All cases have responded to enemas and laxatives. It is recommended that all patients receiving vincristine sulfate follow a routine prophylactic regimen against constipation. Especially in young children, you can bring cases of paralytic ileus (with appearance of surgical abdomen), which are resolved with the temporary suspension of vincristine sulfate and symptomatic treatment.

Genitourinary: Polyuria, dysuria and urinary retention due to bladder weakness. If possible, you discontinue treatment with other drugs that cause urinary retention, particularly in the elderly, during the first days of administration of vincristine sulfate.

Cardiovascular: Hypertension and hypotension. The administration of combinations of chemotherapeutic agents with vincristine to patients who previously received mediastinal irradiation sulfate, has been linked with coronary artery disease and myocardial infarction. It has not been determined causation.

Neurologic: Often neuromuscular adverse reactions are presented sequentially.

At first it can be seen only sensory and paresthesia damage. To continue treatment may occur after neuralgia and motor disorders. It is not described any agent to set aside neuromuscular manifestations associated with treatment with vincristine sulfate.

The administration of vincristine sulfate can exacerbate the symptoms of pre-existing neurological diseases. With continued administration has been observed deep tendon reflexes, foot ptosis, ataxia and paralysis. Diseases of the cranial nerves, isolated paresis and/or paralysis of muscles controlled by cranial motor nerves, can occur without motor disorders elsewhere; generally, the extraocular muscles and laryngeal are the most affected. It has been reported jaw pain, pharyngeal, parotid, bone, back, limb and muscle pain; these pains can be strong. In the small number of patients receiving vincristine sulfate, seizures have been reported, often accompanied by hypertension. In children it has been reported several cases of convulsions followed by coma. They have also been reported cases of transient cortical blindness and optic atrophy with blindness and ocular paralysis. Treatment with vinca alkaloids has resulted rarely damage the eighth cranial nerve that can manifest as vestibular damage (difficulties in the balance including dizziness, nystagmus and vertigo) and hearing damage (partial or total deafness which may be temporary or permanent).

Endocrine: It has been observed in rare cases some patients treated with vincristine sulfate presented the syndrome 
attributed to inadequate secretion of antidiuretic hormone. This syndrome is characterized by high urinary sodium excretion in the presence of hyponatremia; there is kidney disease, adrenal, hypotension, dehydration, azotemia, and clinical edema. With fluid restriction improvement of hyponatremia and renal sodium loss is observed.

Hematologic: vincristine sulfate appears to have no significant effect on constant or platelets or erythrocytes. Severe depression of bone marrow is not usually an important factor limiting the dose. However, there have been cases of anemia, leucopenia and thrombocytopenia. When there thrombocytopenia at the start of treatment with vincristine sulfate, it can improve before marrow remission. Dermatologic: alopecia and skin irritation.

Drug interactions: Simultaneous oral or intravenous administration of phenytoin and antineoplastic combination chemotherapy including vincristine reduced blood levels of anticonvulsant and increased seizure activity. The therapeutic administration of digoxin and vincristine can decrease plasma levels of digoxin and renal excretion.

When administered vincristine with L-asparaginase, it is administered vincristine sulfate 12 to 24 hours before the enzyme to decrease toxicity, since the administration of $\mathrm{L}$-asparaginase before vincristine may decrease hepatic clearance thereof. It should not be given concomitantly with radiotherapy, myelosuppressive drugs or other neurotoxic drugs. Simultaneous use with mitomycin $\mathrm{C}$ can cause acute pulmonary reactions.

\section{Use in pregnancy and lactation}

Pregnancy/lactation: Should not be used during pregnancy especially in the first three months; if necessary use during it, the mother should be advised about the potential risks to the fetus and newborn. It can cause side effects in infants, so it should be discontinued when the drug to the mother is necessary.

Induced neuropathy medical treatment of cancer Antineoplastic agents most frequently related to peripheral neuropathy are taxanes, platinum, vinca alkaloids, epothilones, thalidomide and bortezomib. Currently, there are no preventive or therapeutic interventions to alter the course of the commitment of the peripheral nervous system secondary to these drugs, which often leads to abandonment of cancer treatment and functional impairment.

\section{Factors to boost the risk of neurotoxicity vincristine}

Vinca alkaloid vincristine is the one commonly used chemotherapeutic agent in the treatment of different types of malignancies. Their use may be limited because of peripheral neurotoxicity is dose-related, frequency of administration and patient age.

Vincristine neurotoxicity is well known and characterized with sensorimotor polyneuropathy distal mixed. The toxicity is related to the dose, frequency of administration and the patient's age. The significant neurotoxicity is not generally seen with the cumulative dose, although severe neurotoxicity has been reported after small doses of vincristine in patients with neurological subclinical disease.

McGuire et al. He reported a patient with hereditary neuropathy unacknowledged had sensorimotor polyneuropathy developed severe $3.5 \mathrm{mg}$ after vincristine treatment. They noted general weakness, decreased tendon reflexes and muscle atrophy of the anterior compartment and foot arches prominent. Patients with a family history of hereditary neuropathy. They also analyzed six similar cases in the literature and these cases suggested that there is an increased sensitivity to low doses of vincristine in patients with a pre-existing inherited polyneuropathy. Two similar cases with hereditary polyneuropathy described with development of severe neuropathy after receiving $6 \mathrm{mg}$ vincristine. Most of these cases reported in the literature are treated in children or adolescent patients.

\section{Related cases polyneuropathy and vincristine}

A study with 2 patients in which a case of a patient of 12 years old, diagnosed with liver tumor develops fast foot drop to the left after $1.5 \mathrm{mg} / \mathrm{m}^{2} /$ week intravenous (iv) with reported vincristine treatment. The patient was admitted to the department of neurophysiology on the seventh day of his weakness. In the neurological examination muscle strength of the anterior tibial and peroneal muscles were $0 / 5$ on the left side and the strength of the other muscles of the lower limbs was $4 / 5$ bilaterally. Deep tendon reflexes were reduced at the top and were absent in the lower extremities. He had atrophy in tibialis anterior muscles bilaterally. The examination included electrophysiological nerve conduction studies (NCS) and electromyography. The amplitudes of the ulna and the posterior tibial the PACM were moderately reduced. Latency and conduction velocity of ulnar nerve was normal. The second case was a patient of 15 years of age with a diagnosis of acute myeloid leukemia which received induction chemotherapy, developing bilateral foot drop after 3 weeks of $1.5 \mathrm{mg} / \mathrm{m}^{2} /$ week IV vincristine. The patient was admitted to the department of neurophysiology the sixth day of their symptoms. In the neurological examination the anterior and peroneal tibial muscles were 2/5 bilaterally. The strength of the other muscles was normal. The ROT were absent. He had hammertoes. Electrophysiological examination included only study nerve conduction. The amplitude of the ulna was moderately reduced and the distal latency moderately increased.

\section{Physiopathology}

The mechanism of injury of the peripheral nervous system (PNS) is not known in all cases. Exceptions such as suramin and ara $\mathrm{C}$, chemotherapy-induced neuropathies are axonal. Usually it is associated with the same toxic mechanism on cancer cells. The mechanisms by which chemotherapy induced injury on the SNP can be divided into:

1. Effect on microtubules.

a) Microtubule stabilizing.

b) Inhibition of assembly/disassembly stimulation. 
2. Lesion on neuronal DNA.

3. Inhibition of the proteasome.

4. Induction of autoimmunity.

5. Others.

The toxic effect on the SNP may occur on the neuronal soma or axon. The traditional mechanism of injury is called common to other metabolic, toxic and hereditary neuropathies, retrograde axonal degeneration in which it is assumed that both protein production in the cell body as axonal transport anterograde and retrograde altered.

When the injury occurs strictly on the soma of the sensory neuron talking about neuronopathy or ganglionopathy, such as cisplatin. You can also preferably occur on the motor, sensory or autonomic or be mixed fibers. Some chemotherapy-induced neuropathies predominantly affect the finest fibers, unmyelinated and produce a picture called fine fiber polyneuropathy with clinical and diagnostic peculiarities. Clinical manifestations depend on which type most affected fibers.

The phenomenon of coasting or drag phenomenon is the appearance of new symptoms or progression after discontinuation of the drug. Its pathophysiological mechanism is not well known, but it has been suggested delayed neuronal death by drug binding to mitochondrial DNA and delayed axonal degeneration.

Clinical manifestations the diagnosis of chemotherapy-induced neuropathy can be established clinically. Several severity scales [Rating scales neuropathy] are used. The fastest, cheapest, and effective way of diagnosing chemotherapy-induced neuropathy that is being developed is clinical examination, especially the valuation of reflexes and vibratory sensation, the first signs appear. The motor examination should include assessment of the strength of the extensor hallucis longus muscle in which appreciates the early loss of strength. The symptoms of toxic polyneuropathy by Vincristine.

The list of signs and symptoms mentioned in various sources for toxic polyneuropathy by Vincristine includes the 11 symptoms listed below:

\section{Reduced sensation.}

2. Loss of sensation.

3. Burning sensation.

4. Pain.

5. Numbness.

6. Tingling.

7. Abnormal sensations.

8. Altered sensation.

9. Muscle aches.

10. Muscle weakness.

11. Alteration of movement.

\section{Supplementary Tests}

\section{Electrophysiological-studies}

Studies conventional driving or electroneurography (ENG) are the most reproducible test. However, the ENG is only able to study the thicker fast myelinated fibers. The ENG is essential for the diagnosis of focal neuropathies or plexopathies. It has also been used to identify patients at risk for chemotherapy induced before undergoing chemotherapy neuropathy.

The ENG is neither sensitive nor specific enough, it does not diagnose neuropathies induced by chemotherapy or more incipient affecting the finer fibers. Besides alterations in the ENG usually occur when chemotherapy-induced neuropathy is already established. ENG is probably not necessary for diagnosing chemotherapy-induced neuropathy except in situations where you are atypical clinical manifestations.

The quantitative sensory evaluation techniques allow us to study the functioning of those fibers accounted for ENG, as fine fibers or regional. Studies depend largely on the methodology and patient cooperation. Unfortunately, they are not available in all hospitals.

Axonal excitability techniques are still under development and allow us to study the excitability of the motor and sensory axons. They have been used to study neuropathy oxaliplatin. They could identify patients at increased risk of developing neuropathy irreversible cisplatin.

\section{Biopsy of the skin}

In recent years this technique has been developed to visualize and quantify in vivo nerve fibers in the epidermis. The skin biopsy is being used in some studies of neuroprotection as the gold standard. There is a clinical trial underway to assess the value of skin biopsy in chemotherapy-induced neuropathy.

Treatment: There is at present proved an effective treatment for chemotherapy-induced neuropathy. There is evidence that some nutritional supplements such as L-carnitine could produce improvement in some patients.

Education: Patient education with established chemotherapyinduced neuropathy is essential to improve their quality of life. Should be given adequate information about the need to monitor the temperature of food and objects, use of gloves and socks, the importance of adequate lighting housing, use of canes or braces, anti-slip bathtubs, bars, soap dispensers, maquinillas and electric toothbrushes.

Rehabilitation: Rehabilitation allows many patients improve their quality of life, especially those with proprioceptive disorders. -Symptomatic of neuropathic pain

\section{Clinical Summary}

\section{Hospital outcomes}

It is male patient who is admitted on $12 / 02 / 13$ to present a submandibular and right infraorbital 1 month of evolution was 
managed on an outpatient basis at the health center mass. It was performed 2 weeks before hospitalization in hospital Ocotal lesion biopsy which result mother said that was normal, being referred for assessment by oncology (Table 1).

The $12 / 02 / 13-19 / 02 / 13$ is managed with antibiotics, ceftriaxone and clindamycin. He performed bone aspirate the reporting 2/22/13: Common (B-II) With the diagnosis of acute lymphoblastic leukemia chemotherapy begins.

On 22/02/13 Intrathecal methotrexate, same antibiotic chemotherapy - Intermediate risk pre-phase induction IA.

Home by February 22, 2013. Home Vincristine is given from March 1, 2013. Complying 4 cycles until March 22 On April 2, 2013 presents flaccid paralysis of the lower limbs by using Vincristine.

Physical examination shows muscle weakness and flaccidity of the lower limbs with decreased sensitivity ROT and preserved. It is valued on April 9, 2013 presenting the same picture with lower limb weakness, so neurophysiological studies reporting (25/04/2013) are sent.

Nerve conduction velocity and electromyography of lower limbs: Inductive study of motor axonal polyneuropathy severe type that affects the lower limbs with important active denervation.

Somatosensory evoked potentials lower limb: Somatosensory evoked potentials lower limb with tibial nerve stimulation to within normal limits indicating proper transmission of nerve impulses through posterior spinal cords, medium lemniscus and bilateral thalamocortical radiation.

\section{Methodological design}

Study type: Case study.

Study population: A patient diagnosed with acute lymphoblastic leukemia that developed severe motor polyneuropathy secondary to the use of vincristine.

Area of study: Children's Hospital Manuel de Jesus Rivera, area of hemato-oncolog Source:
Table 1 Male patient outcomes.

\begin{tabular}{|c|c|}
\hline Patient & M.J.G.P \\
\hline Age & 7 years \\
\hline Date of birth & $28-06-2005$ \\
\hline Hometown & Ocotal, Nueva Segovia, Nicaragua \\
\hline Gender & Male \\
\hline Family background & Diabetes maternal uncle \\
\hline medical history & Pain in joints from 2 to 3 years \\
\hline Date & $02-12-2013$ \\
\hline
\end{tabular}

1. Primary physical examination of the patient.

2. Secondary clinical record.

\section{Results}

The study was performed in a patient 7-year-old male from the urban area of Ocotal, without pathological family history and joint pain from approximately 2 years of age. No malformations of the lower limbs. Patient which was entered by the presence of cervical mass and then diagnosed as ALL.

She was treated with vincristine $1.5 \mathrm{mg} / \mathrm{m}^{2} /$ week intravenous (iv) for 4 weeks, in the fifth week showing weakness and flaccidity of muscles of the lower limbs with decreased sensitivity ROT and preserved. It is performed Nerve conduction velocity and electromyography finding lower limb motor axonal polyneuropathy severe type that affects the lower limbs with important active denervation.

\section{Conclusion}

In the present study we found a patient of school age which developing severe motor polyneuropathy secondary axonal type the use of vincristine after 4 weeks of treatment. Meeting at the age of highest incidence according to the literature. It does not show correlation hereditary neuropathy since these patients are more susceptible to using vincristine. The dose and the period of use of vincristine are in the reference range to develop neuropathy from the literature. 


\section{References}

1 Bayrak AO (2007) Severe peripheral neuropathy secondary to vincristine therapy. Department of Neurology, School of Medicine, Ondokuzmayıs University, Samsun, Türkiye.

2 Schiavetti A, Frascarelli M, Uncini S, Novelli A (2004) Vincristine neuropathy: Neurophysiological and Genetic Studies in a case of Wilms tumor. Pediatr Blood Cancer 43: 606-609.

3 McGuire S, Gospe S, Dahl G (1989) Vincristine acute neurotoxicity in the presence of Motor and sensory neuropathy type Med Pediatr Oncol 17: 520-523.
4 Graf WD, Chance PF, Lensch MW, Eng Lipe HP, Bird TD (2001) Severe vincristine neuropathy in Charcot Marie Tooth disease type $1 \mathrm{~A}$. Cancer. nineteen ninety-six 7: 1356-1362.

5 Neumann A, Toren A, Rechavi G, Seifried B, Shoham NG (1996) Vincristine treatment with $M$. Mandel triggering the expression of asymptomatic Charcot Marie Tooth disease. Med Pediatr Oncol 26: 280-283.

6 Orejana-Garcia AM, Pascual-Huerta J, Perez-Melero A (2003) Charcot-Marie-Tooth disease and vincristine. J Am Med Assoc Podiatr 93: 229-233. 